\title{
La disfagia de corta evolución y su asociación con la esofagitis disecante y enfermedades concurrentes. Un reto diagnóstico
}

\author{
Acute dysphagia presentation and its association with esophagitis dissecans and \\ concomitant diseases. A diagnostic challenge
}

\author{
Óscar Teramoto-Matsubara1, Elymir S. Galvis-García ${ }^{2,3,5}$, Juan M. Abdo-Francis, \\ Sergio Sobrino-Cossío ${ }^{2,5 *}$, Antonio Orozco-Gamiz' ${ }^{6}$ Miguel Morales-Arámbula ${ }^{7}$,
} J. Antonio Carrasco-Rojas ${ }^{5}$ y Marisi Meza-Caballero ${ }^{2,8}$

${ }^{1}$ Servicio de gastroenterología, Centro Médico ABC, Ciudad de México; ${ }^{2}$ Centro Avanzado en Endoscopia y Estudios Funcionales, Gástrica, SA de CV, Ciudad de México; ${ }^{3}$ Servicio de Endoscopia, Hospital General de México Dr. Eduardo Liceaga; ${ }^{4}$ Servicio de Endoscopia, Hospital Ángeles Acoxpa, Ciudad de México; ${ }^{5}$ Servicio de Endscopia, Hospital Ángeles del Pedregal. Ciudad de México; ${ }^{6}$ Servicio de Endoscopia, Gastrolab Laboratorio de Fisiología Gastrointestinal, Guadalajara, Jalisco; ${ }^{7}$ Servicio de Gastroenterología, Hospital Country 2000, Guadalajara, Jalisco; ${ }^{8}$ Centro Privado de Anatomía Patológica y Citología Exfoliativa, Ciudad de México. México

\begin{abstract}
Resumen
La esofagitis disecante (ESD) es una enfermedad rara que puede asociarse a esofagitis eosinofílica, candidiásica o medicamentos. Se analizaron las características clinico-endoscópicas e histopatológicas en sujetos con disfagia de corta evolución asociada a afecciones concurrentes. Estudio observacional de cohorte retrospectiva con disfagia y daño mucoso. Se ingresaron 23 pacientes con edad media de 55 años. Todos tuvieron síntomas de reflujo gastroesofágico (ERGE), disfagia y odinofagia. Fueron 10, 9 y 4 casos de ESD, descamativa/esfacelante (ESD/ES) y fibroestenosis, respectivamente. La ESD/SE es una afección rara, con un espectro clínico, endoscópico e histológico variable.
\end{abstract}

PALABRAS CLAVE: Candidiasis esofágica. Disfagia. Esofagitis disecante. Esofagitis eosinofílica.

\begin{abstract}
Esophagitis dissecans (ESD) is an uncommon disease that is associated with eosinophilic esophagitis (EoE), candidiasis and/ or drugs. We aim to characterize the clinical, endoscopic and histological damage of the mucosal in subjects with dysphagia, its relation to concomitant entities and the treatment response. This is a retrospective observational study in patients with dysphagia and esophageal mucosal damage We included 23 patients (mean age = 55 years) who had GERD symptoms, dysphagia and/or odynophagia. There were 10, 9 and 4 cases of esophagitis dissecans, desquamative/esfacelante (ESD/ES) and fibrostenotic, respectively. ESD/SE is a rare entity with variable clinical, endoscopic and histological spectrum. The association with GERD was frequent.
\end{abstract}

KEY WORDS: Esophageal candidiasis. Dysphagia. Esophagitis dissecans. Eosinophilic esophagitis.

\author{
Correspondencia: \\ *Sergio Sobrino-Cossío \\ Camino a Santa Teresa, 1055-776 \\ Col. Héroes de Padierna \\ C.P. 10700 , Ciudad de México, México \\ E-mail: ssobrinocossio@gmail.com
}

Fecha de recepción: 23-07-2018

Fecha de aceptación: 10-11-2018

DOI: $10.24875 / C I R U .18000607$
Cir Cir. 2019;87:572-579

Contents available at PubMed www.cirugiaycirujanos.com 


\section{Introducción}

La barrera epitelial esofágica protege a las capas profundas de la mucosa y la submucosa del contenido nocivo intraluminal'. La reparación tisular es consecuencia de la migración de las células viables adyacentes hacia las zonas necróticas ${ }^{2,3}$.

Los mecanismos de daño difieren en la enfermedad por reflujo gastroesofágico (ERGE). En la variedad erosiva, el daño es por contacto del ácido sobre la mucosa, aumento de la dilatación de los espacios intercelulares y su permeabilidad, consecuentemente por el transporte paracelular de hidrogeniones ${ }^{4-7}$. La exposición crónica al reflujo patológico y la falla del mecanismo de regeneración tisular son causales de la esofagitis erosiva1.

Por otra parte, la variedad no erosiva es consecuencia de un proceso inflamatorio generalizado (citocinas proinflamatorias interleucinas 8 y $1 \beta$ ) iniciado dentro de la capa basal y se caracteriza por el aumento de linfocitos intraepiteliales, hiperplasia de las glándulas basales e hipertrofia papilar ${ }^{8-10}$.

La pirosis es un evento intraesofágico que no necesariamente está relacionado con la exposición al ácido. La magnitud del daño tisular no se correlaciona con la intensidad de los síntomas-ERGE ${ }^{8}$. Existen varias enfermedades que producen daño de la mucosa.

Los criterios diagnósticos clínico-patológicos ${ }^{11}$ de la esofagitis eosinofílica establecidos son: 1) síntomas asociados a disfunción esofágica; 2) infiltrado inflamatorio por eosinófilos (> 15 eosinófilos por campo de alto poder); 3) eosinofilia persistente tras la utilización de un inhibidor de la bomba de protones (IBP); 4) exclusión de otras causas de eosinofilia; y 5) respuesta al tratamiento (dieta de eliminación, corticoesteroides tópicos) $)^{11,12}$.

La apariencia endoscópica de la esofagitis eosinofílica es variable, desde anillos concéntricos, placas blanquecinas y surcos hasta el esófago de pequeño calibre $^{13,14}$. El mecanismo de la disfagia es multifactorial, destacándose rigidez de la mucosa, pérdida de la distensibilidad, fibrosis, estenosis y anillos ${ }^{15-19}$. La prevalencia se estima en $0.5-1$ en 1000 , y es la principal causa de impactación de alimento en el esófago ${ }^{20}$.

La esofagitis medicamentosa es frecuente en la edad avanzada y se asocia al consumo de múltiples fármacos, ingestión de poca cantidad de líquido y peristalsis fallida ${ }^{21}$. Los antibióticos, los antiinflamatorios no esteroideos (AINE), los bisfosfonatos, el potasio de liberación lenta y los suplementos de hierro son los fármacos que con mayor frecuencia causan daño ${ }^{22}$.

Por otra parte, la candidiasis es una infección oportunista que puede infectar áreas de inflamación y ulceración ${ }^{23-25}$, y concurrir con la esofagitis disecante $(12.9 \%)^{26,27}$. Si existe infiltración neutrofílica deberá descartarse la infección fúngica superpuesta ${ }^{28}$.

Aunque algunos autores las han considerado como una sola afección ${ }^{27}$, la esofagitis disecante superficial (EDS) y la esofagitis esfacelante o descamativa (SE) comparten características endoscópicas e histopatológicas ${ }^{28}$. La ESD/SE es una condición benigna, de la que se han reportado poco menos de 200 casos en la literatura ${ }^{29,30}$. La causa es idiopática, aunque hay reportes posradioterapia y asociada a la enfermedad autoinmunitaria del injerto contra el huésped ${ }^{31}$.

El traumatismo químico por fármacos (antidepresivos, AINE y bisfosfonatos) ${ }^{33-35}$ y térmico-físico por bebidas calientes, irritantes químicos y escleroterapia de varices, puede dañar la mucosa ${ }^{36}$. La presentación clínica es variable, en ocasiones con la expulsión de grandes costras de mucosa ${ }^{37-39}$. Se ha asociado a la dermatitis bullosa autoinmunitaria (pénfigo/penfigoide $)^{37-40}$, la enfermedad celíaca ${ }^{41}$ y el tabaquismo crónico ${ }^{27}$. El espectro del daño varía desde áreas de descamación epitelial, fisuras verticales y la esfacelación del epitelio superficial blanquecino ${ }^{32}$ hasta el esófago tubular ${ }^{42}$. Otros hallazgos endoscópicos son eritema, nodularidad, anillos e incluso estenosis ${ }^{43,44}$. La histología puede mostrar descamación del epitelio escamoso superficial con separación de las capas, paraqueratosis, necrosis coagulativa e inflamación aguda o crónica ${ }^{45}$.

Es complejo saber si los síntomas-ERGE son una causa $o$ un efecto, o si tienen un efecto aditivo o multiplicativo sobre la expresión de la enfermedad. La presentación clínica y los hallazgos endoscópicos e histopatológicos son muy variables. La respuesta al tratamiento dependerá del diagnóstico adecuado; sin embargo, la probabilidad de estar frente a situaciones clínicas concurrentes y superpuestas es muy alta, lo cual representa un verdadero reto de diagnóstico.

El objetivo del estudio fue caracterizar casos de EDS/SE desde el punto de vista clínico, endoscópico e histopatológico, y evaluar su respuesta al tratamiento (cicatrización de la mucosa) en sujetos con disfagia de corta evolución asociada a otras condiciones concurrentes (esofagitis eosinofílica, medicamentosa 0 candidiásica) en sujetos con síntomas-ERGE. 


\section{Método}

Estudio descriptivo y observacional de una cohorte retrospectiva de sujetos con disfagia de corta evolución y daño de la mucosa (esofagitis disecante, descamativa o esfacelante) evaluados por endoscopia en un centro privado de la Ciudad de México del 1 de 2009 al 31 de diciembre 2017.

Se investigó en la base de datos del archivo endoscópico y se seleccionaron aquellos con el diagnóstico endoscópico/histológico de esofagitis disecante, esfacelante o descamativa, medicamentosa o eosinofílica, con candidiasis esofágica o fibroestenosis esofágica.

Cada reporte endoscópico, fotografías y videos se revisaron en forma independiente por dos investigadores y se seleccionaron ante la sospecha diagnóstica de las afecciones mencionadas. Las discrepancias en el diagnóstico fueron solucionadas mediante la discusión de los casos, y si fue necesario mediante la intervención de un tercer investigador. Se revisó el archivo de patología en búsqueda de los diagnósticos de esofagitis disecante, esofagitis disecante superficialis o esofagitis esfacelante, esofagitis eosinofílica y esofagitis por cándida ${ }^{26}$.

Se evaluaron las características clínicas, el tiempo de evolución de los síntomas hasta el momento de la endoscopia y las indicaciones para el procedimiento. Se revisaron las notas clínicas sobre uso de medicamentos y consumo de bebidas calientes los días previos al inicio de los síntomas.

En ausencia de criterios diagnósticos para ESD, Hart, et al. ${ }^{26}$ generaron una lista a priori de características endoscópicas basado en su experiencia. Los tres criterios requeridos fueron: 1) tira(s) de mucosa esofágica desprendida(s) $>2 \mathrm{~cm}$ de longitud; 2) mucosa esofágica subyacente normal; y 3) falta de ulceraciones o friabilidad de la mucosa esofágica adyacente. Se reportaron las características endoscópicas e histopatológicas con la tinción de hematoxilina-eosina y ácido periódico de Schiff (PAS).

Se evaluaron la respuesta sintomática y la cicatrización de la mucosa después del tratamiento con IBP con un seguimiento de 6 meses.

\section{Estadística}

Estadística descriptiva con medidas de tendencia central (media y mediana). Los datos fueron presentados como frecuencia y proporciones.

\section{Resultados}

Se ingresaron 23 pacientes con edad promedio de 55 años (rango: $18-92)$; el $65.2 \%$ eran mujeres. Todos los pacientes refirieron pirosis de intensidad variable (17 moderada y 6 grave). La aparición de la disfagia fue la principal indicación para la endoscopia. Dos casos manifestaron afagia. El diagnóstico endoscópico fue de esofagitis disecante en 10, descamativa 0 esfacelante en 9 y fibroestenosis en 4 casos.

La ESD/ES fue más frecuente (70\%) en los mayores de 40 años y el tiempo de evolución de los síntomas osciló entre 7 y 88 días (mediana: 25.5 días; rango: 7-88 días).

Entre los antecedentes de importancia se encontró que dos casos reportaron tabaquismo crónico intenso, cinco osteoartritis, cuatro hipotiroidismo, dos diabetes mellitus tipo 2, dos hipertensión arterial, uno insuficiencia renal terminal y otro más cirrosis hepática con Child B sin varices esofágicas. Dos tuvieron coronariopatía compensada e ingestión crónica de antiagregantes plaquetarios y ácido acetilsalicílico por el antecedente de cateterismo coronario previo con fracciones de expulsión del $59 \%$ y el $66 \%$, respectivamente. Uno más recibió quimioterapia por cáncer colorrectal. Ningún paciente tuvo lesiones de la piel ni enfermedad autoinmunitaria.

La tabla 1 resume el consumo de medicamentos. El tiempo entre la ingestión de AINE y la aparición de disfagia fue menor de 7 días en tres casos (13\%). Un paciente con politraumatismo reportó síntomas durante la toma de múltiples fármacos (penicilina, dicloxacilina, clindamicina, tramadol, paracetamol e ibuprofeno $600 \mathrm{mg} / \mathrm{día}$ ). Dos la asociaron con la ingesta de bebidas muy calientes y uno más con la mostaza rusa.

La respuesta clínica (síntomas-ERGE) al IBP por vía oral (40 mg/día) mediante prescripción médica o automedicación previa a la endoscopia fue insatisfactoria en todos los casos.

Otros síntomas fueron pirosis $(100 \%)$, odinofagia $(82,6 \%)$, regurgitación $(62,1 \%)$, pérdida de peso $(21,7 \%)$, dolor torácico no cardíaco $(17,4 \%)$ y hematemesis (un caso con afagia). Dos casos tuvieron impactación de alimento y dos casos de medicamento.

La endoscopia fue ambulatoria en el $75 \%$ de los casos. El esófago medio y el distal fueron los segmentos más afectados, y en un solo caso (hematemesis) la esfacelación se localizó en el segmento proximal (Fig. 1). 


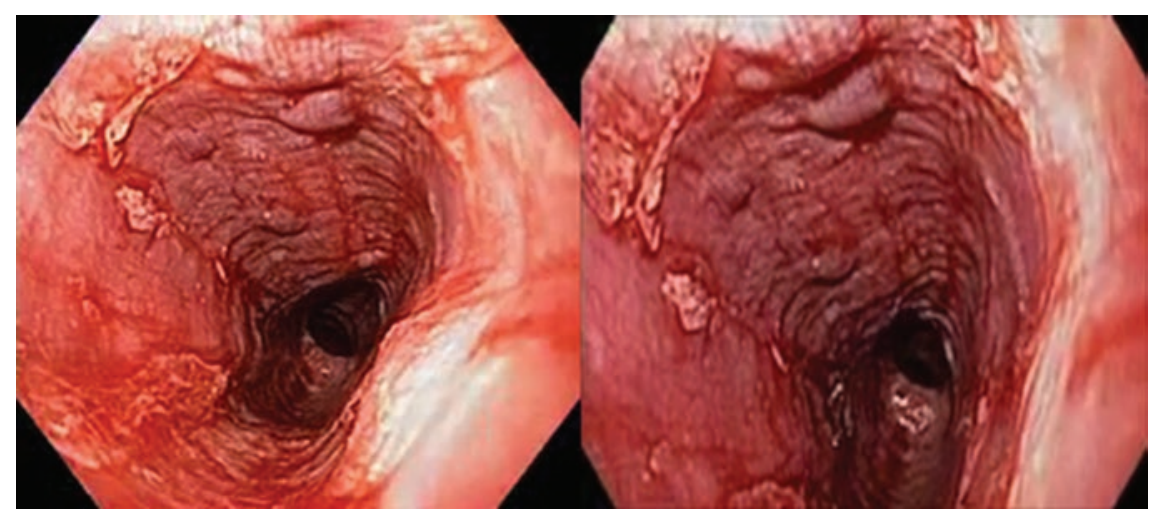

Figura 1. Esófago con áreas de esfacelación y hemorragia rezumante en paciente con impactación de medicamento en el esófago proximal.

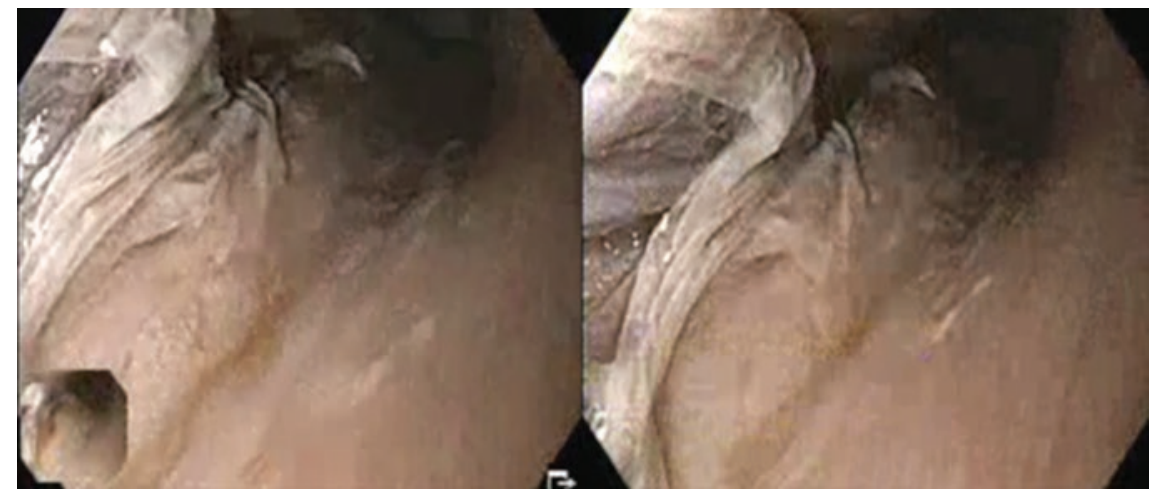

Figura 2. Esófago medio con áreas extensas de esfacelación de la mucosa y bulla.

Tabla 1. Fármacos asociados a daño agudo de la mucosa esofágica en una cohorte de disfagia aguda o subaguda

\begin{tabular}{llr}
\hline Medicamentos & $\mathbf{n}$ & $\%$ \\
\hline Antiinflamatorios no esteroideos & 12 & 52.1 \\
Ácido acetilsalicílico (81 o $325 \mathrm{mg}$ ) & 8 & 34.8 \\
Suplementos de potasio o calcio & 4 & 17.4 \\
Cloruro de potasio o hierro oral & 4 & 17.4 \\
$\begin{array}{l}\text { Inhibidores selectivos de la recaptura de serotonina o } \\
\text { noradrenalina }\end{array}$ & 4 & 17.4 \\
\hline
\end{tabular}

Los hallazgos endoscópicos fueron inflamación (12 casos), friabilidad (10), descamación (9), edema (6), ulceración (5), estenosis (4), anillos (4) y nodularidad (4). La esfacelación o descamación estuvo presente en 19 casos, 10 de ellos con extensas áreas de denudación y desprendimiento de la superficie epitelial y zonas de necrosis (Figs. 2 a 5).

En tres pacientes la ESD/SE fue concurrente con candidiasis (confirmación citológica), observándose placas blanquecinas confluentes, exudado y desprendimiento de tiras de mucosa.

En cuatro casos se confirmó el diagnóstico endoscópico e histopatológico de esofagitis eosinofílica (dos de ellos con fibroestenosis). En dos casos se observó estenosis esofágica y anillos concéntricos (traquealización o esófago felino), y en otros dos hubo placas blanquecinas confluentes y líneas de surcos.

La histopatología se realizó en 18 (78.2\%) casos, reportándose paraqueratosis (100\%) e hiperplasia de células basales (75\%) (Fig. 6). En la mitad de los casos se reportó infiltrado inflamatorio focal con actividad por neutrófilos, el $22.2 \%$ eosinófilos y el $16.6 \%$ linfocitos, y en el resto no hubo evidencia de respuesta inflamatoria. En ellos, el epitelio escamoso superficial tenía aspecto «momificado» y se observaron bullas en dos casos. No se reportaron alteraciones dentro de la capa basal.

Los casos con impactación de alimento (carne de pollo) se trataron satisfactoriamente mediante la remoción endoscópica desde el esófago medio y distal, con altas dosis de IBP intravenoso y ayuno.

Un paciente presentó afagia y hematemesis súbitas tras la ingestión de una cápsula de bromuro de pinaverio (100 mg) y otro solo afagia con AINE (ibuprofeno, $400 \mathrm{mg}$ ). Ambos casos fueron tratados conservadoramente, con buena respuesta clínica a altas dosis de IBP. 


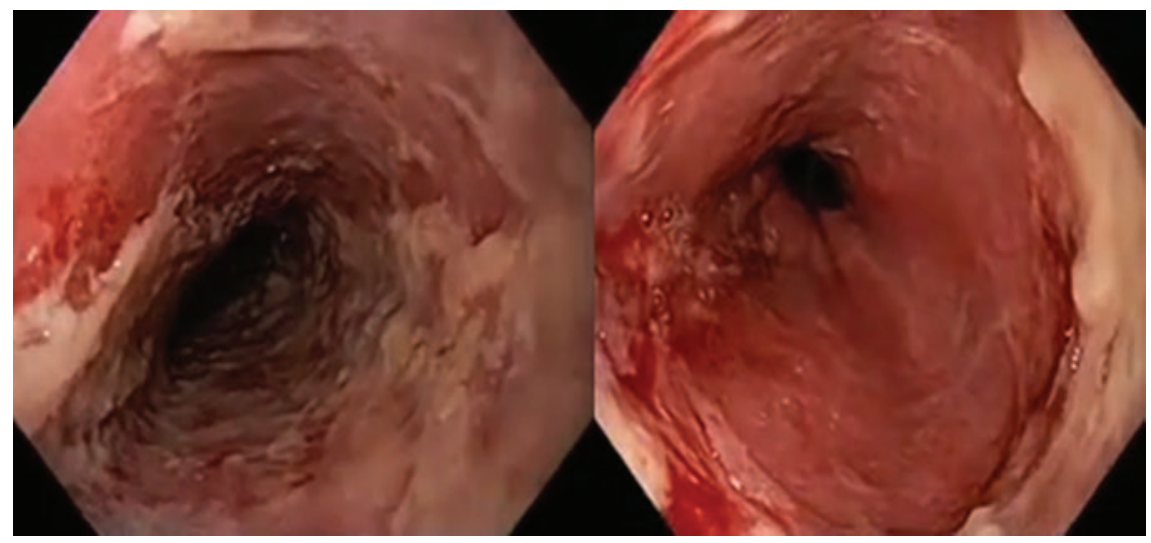

Figura 3. Esófago con áreas extensas denudadas en el esófago medio.

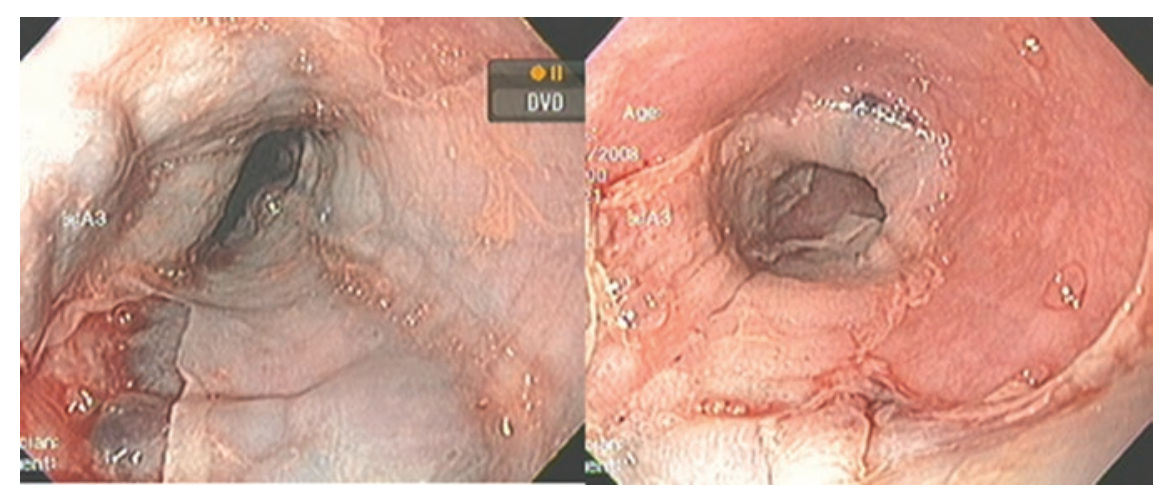

Figura 4. Esófago con áreas extensas de desprendimiento del epitelio y zonas denudadas.

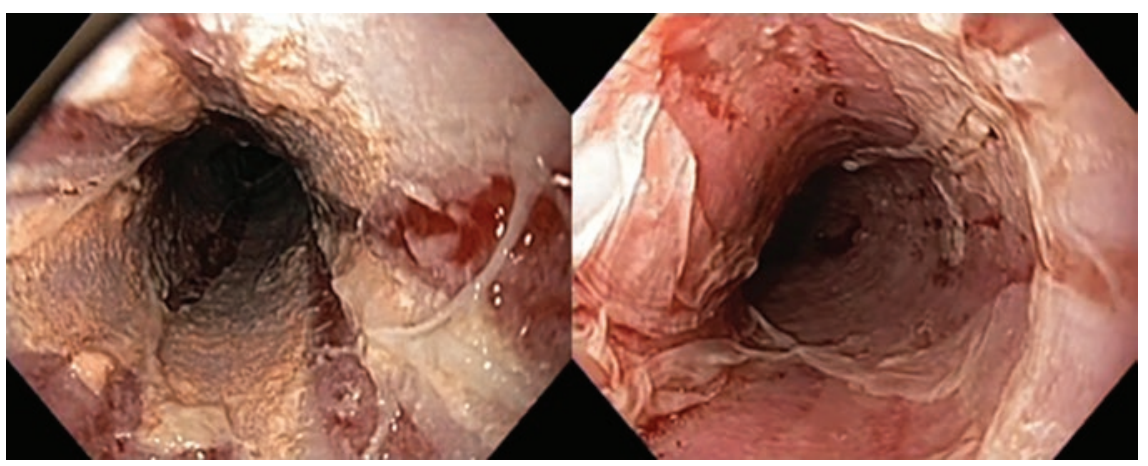

Figura 5. Características típicas de esofagitis dissecans superficialis con grandes áreas de desprendimiento del epitelio escamoso superficial y áreas denudadas.

La respuesta clínica (sintomática) y endoscópica (cicatrización de la mucosa) en la ESD/SE fue satisfactoria tras la toma de IBP, $40 \mathrm{mg}$ dos veces al día durante 8 semanas, en 17 de 19 casos (89.4\%); en aquellos con ESD/SE más candidiasis esofágica concurrente, la respuesta fue parcial tras el IBP y fluconazol.

La mejoría de la disfagia posterior al uso de esteroides fue parcial en los casos de fibroestenosis. Dos casos con esofagitis eosinofílica más candidiasis fueron tratados con esteroides y fluconazol, con mejoría parcial (disfagia intermitente) en el seguimiento clínico.
En el control endoscópico (2-6 meses) se observó la cicatrización de la mucosa en todos los casos, aunque sin la resolución del estrechamiento esofágico. Durante el seguimiento a 6 meses fallecieron tres pacientes, dos con cardiopatía coronaria y uno con diabetes mellitus descompensada e insuficiencia renal.

\section{Discusión}

La esofagitis disecante o esfacelante tiene características clínicas, endoscópicas e histológicas 


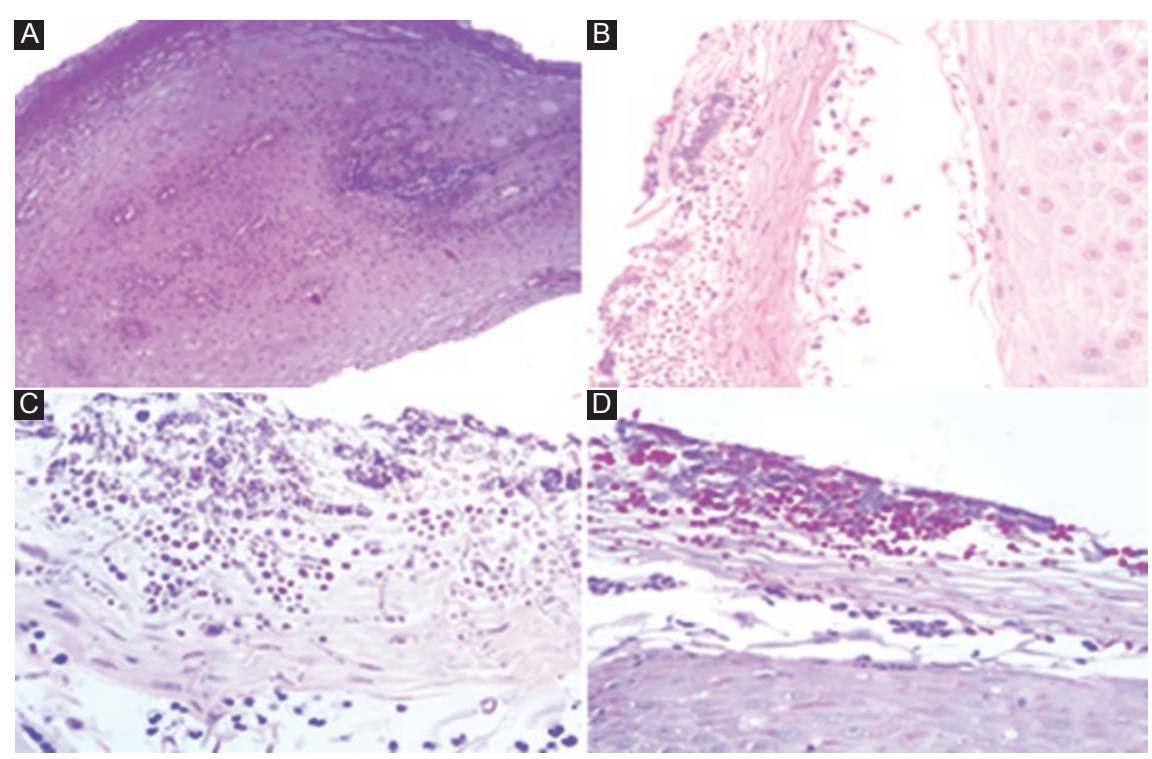

Figura 6. A: Se observa epitelio esofágico que muestra acantosis e hiperplasia de las células basales. B, C y D: Presencia de microorganismos conformados por hifas y esporas positivos a la tinción con PAS, compatibles con Candida sp.

variables. La presencia de síntomas-ERGE y el uso de fármacos 0 bebidas irritantes fueron los factores detonantes asociados a la disfagia.

La exposición al reflujo patológico y la falla en el mecanismo de regeneración epitelial causan el daño de la mucosa esofágica'. La eosinofilia intramucosa es frecuente en la ERGE y requiere efectuar un diagnóstico diferencial con patologías como la esofagitis eosinofílica ${ }^{11}$. Por otra parte, la infección fúngica (candidiasis) puede ser concurrente y no la causa de la enfermedad ${ }^{23}$. El tipo de diseño metodológico de nuestro estudio no permitió establecer los criterios de causalidad.

Una característica clínica, antes de la aparición de disfagia, fue la nula o parcial respuesta al IBP de los síntomas-ERGE. Aunque los mecanismos fisiopatológicos entre las variedades erosiva ${ }^{4-7}$ y no erosiva ${ }^{8-10}$ difieren, consideramos que en esta existe la superposición de daño de la mucosa y proceso inflamatorio generalizado subyacente debido a la gran expresión de la enfermedad.

Las principales características del daño histopatológico en la magnitud del daño, I es, y la inmunosupresie daño de la mucosa.ralizado.s de hierro, an una sola entidad.os entógico en la ERGE son: 1) hiperplasia de las células basales (grosor > 15\%); 2) hipertrofia papilar (> 50\%); 3) inflamación intraepitelial, incluidos eosinófilos; y 4) edema intercelular (espongiosis). Si el número de eosinófilos es elevado deberá descartarse liquen plano o esofagitis eosinofílica ${ }^{8}$. La superposición de factores (ERGE, tabaquismo, drogas, bebidas calientes e inmunosupresión) puede tener un efecto sinérgico y favorecer el daño $0^{8-10}$. La mayoría de estos cambios histopatológicos fueron confirmados en nuestra serie.

Es muy difícil ponderar cada factor predisponente (intensidad-ERGE, polifarmacia, comorbilidad, etc.) en condiciones clínicas de baja prevalencia. Consideramos que la presencia de factores detonantes (fármacos, ingestión de bebidas calientes o irritantes) en sujetos susceptibles (ERGE crónica) es un requisito indispensable para su aparición.

Aunque no existen características histológicas específicas de la $\mathrm{ERGE}^{8}$, la infiltración por eosinófilos y linfocitos fue una constante en la mayoría de los casos, mientras que solo en cuatro casos se confirmó el diagnóstico de esofagitis eosinofílica.

La pirosis se debe a un evento intraesofágico que no necesariamente está relacionado con la exposición esofágica al ácido. La percepción del síntoma puede conducir al error diagnóstico. La intensidad sintomática no es dependiente del grado de daño de la mucosa, por lo que es complejo analizar si existen efectos aditivos (sinérgicos) o multiplicativos que expliquen la magnitud de la enfermedad.

En nuestra serie, los casos de impactación por alimento o medicamento fueron resueltos satisfactoriamente. La impactación es una forma de presentación de la esofagitis eosinofílica y es consecuencia de la estenosis $^{13}$. La endoscopia tiene baja sensibilidad y poca especificidad diagnóstica para evaluar la estenosis $^{15}$. La fibroestenosis contribuye a la disfagia ${ }^{16-18}$ y también puede asociarse a dismotilidad esofágica ${ }^{19}$. El desprendimiento de las células escamosas 
superficiales también puede verse en esta enfermedad y responde al IBP.

En sujetos sanos, Candida albicans es un colonizador común del esófago que puede infectar áreas de inflamación y ulceración producidas por otras afecciones $^{24,25}$. Las esporas y las hifas se localizan en la queratina descamada, el material fibrinopurulento o el epitelio escamoso ${ }^{24,26}$. La candidiasis puede sospecharse ante la presencia de placas amarillas 0 blanquecinas sobre la mucosa. Sin embargo, puede presentarse en la esofagitis medicamentosa, la esofagitis eosinofílica y la infección superpuesta. Los linfocitos intraepiteliales pueden simular pseudohifas y la candidiasis puede ser concurrente con ESD (12.9\%).

La infección micótica se sospecha ante la presencia de infiltrado por neutrófilos y falla de respuesta a los IBP. En nuestra serie, tres pacientes tuvieron EDS/SE asociada a candidiasis.

Los medicamentos pueden causar patrones de daño diversos. La historia clínica facilita el diagnóstico diferencial ${ }^{38,39}$. Aunque el daño es más frecuente en edades avanzadas y predomina en el esófago distal 0 en el esófago medio (arco aórtico) ${ }^{22,23}$, en nuestra serie el rango de edad fue muy amplio y el $30 \%$ fueron menores de 40 años. La ingesta insuficiente de líquido o la posición supina predisponen a la retención de la droga. La mucosa, en ocasiones, puede tener la apariencia de un exudado semejante a la candidiasis esofágica ${ }^{38-40}$.

La ESD/SE se ha asociado a la dermatitis bullosa autoinmunitaria (pénfigo/penfigoide) ${ }^{38}$. Los penfigoides son enfermedades autoinmunitarias de la membrana mucosa con afectación cutánea y mucosa (oral, ocular, nariz y faringe, genital, anal y esofágica $)^{40}$. Otras asociaciones reportadas son la enfermedad celíaca ${ }^{41}$ y el tabaquismo crónico intenso ${ }^{27}$.

La descamación total con áreas denudadas, largas roturas lineales de la mucosa, fisuras verticales y grietas circunferenciales con descamación, mucosa blanquecina con hemorragia y esofagitis exudativa, son algunas de las características reportadas ${ }^{37}$. El epitelio escamoso superficial se transforma en necrótico y la mucosa toma una apariencia bitonal (paraqueratosis) debido al contraste entre el tejido necrótico y las células escamosas viables ${ }^{28}$.

La mucosa puede desprenderse con facilidad a la toma de biopsias (signo endoscópico de Nikolsky) ${ }^{37}$. La obtención de material adecuado (membrana basal) se dificulta por la posición tangencial del endoscopio y las pinzas en relación con el esófago ${ }^{37}$.
El diagnóstico de esta enfermedad es endoscópico e histopatológico ${ }^{32,44}$. En nuestra serie, la paraqueratosis, la hiperplasia de las células basales y el epitelio escamoso superficial «momificado» fueron los signos más constantes.

El espectro del daño de la mucosa asociado a la ERGE es amplio y variable. La presencia de afecciones superpuestas en individuos susceptibles expuestos a factores detonantes representa un reto diagnóstico, ya que la expresión de la enfermedad está amplificada.

\section{Conclusiones}

La ESD/SE es una enfermedad infrecuente, con un espectro clínico, endoscópico e histológico variable. La asociación con ERGE es constante. La disfagia fue la principal indicación para la endoscopia. Esta enfermedad probablemente represente la superposición de diferentes condiciones clínicas que dificultan el diagnóstico.

\section{Conflicto de intereses}

Los autores declaran no tener conflicto de intereses.

\section{Responsabilidades éticas}

Protección de personas y animales. Los autores declaran que para esta investigación no se han realizado experimentos en seres humanos ni en animales.

Confidencialidad de los datos. Los autores declaran que han seguido los protocolos de su centro de trabajo sobre la publicación de datos de pacientes.

Derecho a la privacidad y consentimiento informado. Los autores han obtenido el consentimiento informado de los pacientes y/o sujetos referidos en el artículo. Este documento obra en poder del autor de correspondencia.

\section{Bibliografía}

1. Blevins $\mathrm{CH}$, lyer PG, Vela MF, Katzka DA. The esophageal epithelial barrier in health and disease. Clin Gastroenterol Hepatol. 2018;16:608-17.

2. Jiménez $P$, Lanas $A$, Piazuelo $E$, et al. Effect of growth factors and prostaglandin E2 on restitution and proliferation of rabbit esophageal epithelial cells. Dig Dis Sci. 1998;43:2309-16.

3. Jiménez $P$, Lanas A, Piazuelo $E$, et al. Effects of extracellular $\mathrm{pH}$ on restitution and proliferation of rabbit oesophageal epithelial cells. Aliment Pharmacol Ther. 1999;13:545-52.

4. Shen L. Tight junctions on the move: molecular mechanisms for epithelial barrier regulation. Ann N Y Acad Sci. 2012;1258:9-18.

5. Souza RF, Huo X, Mittal V, et al. Gastroesophageal reflux might cause esophagitis through a cytokine-mediated mechanism rather than caustic acid injury. Gastroenterology. 2009;137:1776-84. 


\section{O. Teramoto-Matsubara, et al.: Disfagia y esofagitis disecante}

6. Honda J, lijima K, Asanuma K, et al. Estrogen enhances esophageal barrier function by potentiating occludin expression. Dig Dis Sci. 2016;61:1028-38

7. Álvaro-Villegas JC, Sobrino-Cossío S, Hernández-Guerrero A, et al. Dilated intercellular spaces in subtypes of gastroesophagic reflux disease. Rev Esp Enferm Dig. 2010;102:302-7.

8. Green A, Streutker CJ. Esophagitis: old histologic concepts and new thoughts. Arch Pathol Lab Med. 2015;139:723-9.

9. Krugmann J, Neumann $\mathrm{H}$, Vieth M, Armstrong D. What is the role of endoscopy and oesophageal biopsies in the management of GERD? Best Pract Res Clin Gastroenterol. 2013;27:373-85.

10. Fiocca R, Mastracci L, Riddell R, et al. Development of consensus guidelines for the histologic recognition of microscopic esophagitis in patients with gastroesophageal reflux disease: the Esohisto project. Hum Pathol. 2010;41:223-31.

11. Dellon ES, Gonsalves N, Hirano I, Furuta GT, Liacouras CA, Katzka DA ACG clinical guideline: evidenced based approach to the diagnosis and management of esophageal eosinophilia and eosinophilic esophagitis (EoE). Am J Gastroenterol. 2013;108:679-92.

12. Hirano I, Aceves SS. Clinical implications and pathogenesis of esophageal remodeling in eosinophilic esophagitis. Gastroenterol Clin North Am. 2014:43:297-316.

13. Parfitt JR, Gregor JC, Suskin NG, Jawa HA, Driman DK. Eosinophilic esophagitis in adults: distinguishing features from gastroesophageal reflux disease: a study of 41 patients. Mod Pathol. 2006;19:90-6.

14. Gentile N, Katzka D, Ravi K, Trenkner S, Enders F, Killian J, et al. Oesophageal narrowing is common and frequently under-appreciated at endoscopy in patients with oesophageal eosinophilia. Aliment Pharmacol Ther. 2014:40:1333-40.

15. Lee J, Huprich J, Kujath C, et al. Esophageal diameter is decreased in some patients with eosinophilic esophagitis and might increase with topical corticosteroid therapy. Clin Gastroenterol Hepatol. 2012;10:481-6.

16. Kwiatek MA, Hirano I, Kahrilas PJ, Rothe J, Luger D, Pandolfino JE. Mechanical properties of the esophagus in eosinophilic esophagitis. Gastroenterology. 2011;140:82-90.

17. Moawad FJ, Robinson CL, Veerappan GR, Summers TA Maydonovitch CL, Wong RKH. The tug sign: an endoscopic feature of eosinophilic esophagitis. Am J Gastroenterol. 2013;108:1938-9.

18. Chehade M, Sampson HA, Morotti RA, Magid MS. Esophageal subepithelial fibrosis in children with eosinophilic esophagitis. J Pediatr Gastroenterol Nutr. 2007;45:319-28.

19. Liacouras CA, Furuta GT, Hirano I, et al. Eosinophilic esophagitis: updated consensus recommendations for children and adults. J Allergy Clin Immunol. 2011;128:3-20.

20. Dellon ES. Epidemiology of eosinophilic esophagitis. Gastroenterol Clin North Am. 2014;43:201-18

21. Tutuian R. Adverse effects of drugs on the esophagus. Best Pract Res Clin Gastroenterol. 2010;24:91-7.

22. De Petris G, Gatius Caldero S, Chen L, et al. Histopathological changes in the gastrointestinal tract due to drugs: an update for the surgical pathologist (part I of II). Int J Surg Pathol. 2014;22:120-8.

23. Wilcox CM, Schwartz DA. Endoscopic-pathologic correlates of Candida esophagitis in acquired immunodeficiency syndrome. Dig Dis Sci. 1996;41:1337-45

24. Maguire A, Sheahan K. Pathology of oesophagitis. Histopathology. 2012;60:864-79.
25. Almashat SJ, Duan L, Goldsmith JD. Non-reflux esophagitis: a review of inflammatory diseases of the esophagus exclusive of reflux esophagitis. Sem Diagn Path. 2014;31:89-99.

26. Hart PA, Romano RC, Moreira RK, Ravi K, Sweetser S. Esophagitis dissecans superficialis: clinical, endoscopic, and histologic features. Dig Dis Sci. 2015;60:2049-57.

27. Carmack SW, Vemulapalli R, Spechler SJ, Genta RM. Esophagitis dissecans superficialis ("sloughing esophagitis"): a clinicopathologic study of 12 cases. Am J Surg Pathol. 2009;33:1789-94.

28. Purdy JK, Appelman HD, McKenna BJ. Sloughing esophagitis is associated with chronic debilitation and medications that injure the esophageal mucosa. Mod Pathol. 2012;25:767-75.

29. Ma Ch, et al. Recently highlighted non-neoplastic pathologic entities of their upper Gl tract and their clinical significance. Gastrointest Endosc. 2014;80:960-5.

30. Rosenberg B. Oesophagitis dissecans superficialis. Centralbl Allg Pathol u Path Anat. 1892;3:753-9.

31. Chen WC, et al. Desquamative esophagitis: a refractory mucosal injury pattern. Dis Esophagus. 2017;30:1-5

32. Abbass K, Haveman L, Gertner E. Esophagitis dissecans superficialis due to severe methotrexate toxicity. Endoscopy. 2014;46(Suppl 1): E99-100.

33. Cameron RB. Esophagitis dissecans superficialis and alendronate: case report. Gastrointest Endosc. 1997;46:562-3.

34. Hokama A, Ihama Y, Nakamoto M, Kinjo N, Kinjo F, Fujita J. Esophagitis dissecans superficiales associated with bisphosphonates. Endoscopy. 2007;39(Suppl 1):E91.

35. De Petris G, Gatius Caldero S, et al. Histopathological changes in the gastrointestinal tract due to medications: an update for the surgical pathologist (part II of II). Int J Surg Pathol. 2013;22:202-11.

36. Pérez-Carreras M, Castellano G, Colina F, et al. Esophagitis dissecans superficialis (esophageal cast) complicating esophageal sclerotherapy. Am J Gastroenterol. 1998:93:655-6.

37. Hokama A, Yamamoto Y, Taira K, et al. Esophagits dissecans superficialis and autoimmune bullous dermatoses: a review. World J Gastrointest Endosc. 2010;2:252-6.

38. Shih A, Misdraji J. Drug-induced pathology of the upper gastrointestinal tract. Diagnostic Histopathology. 2017;23:84-95.

39. Zografos GN, Georgiadou D, Thomas D, et al. Drug-induced esophagitis. Dis Esophagus. 2009;22:633e7.

40. Zehou O, Raynaud JJ, Roux-Villet L, Alexandre M, Airinei G, Pascal F, et al. Oesophageal involvement in 26 consecutive patients with mucous membrane pemphigoid. Br J Dermatol. 2017;177:1074-85.

41. Hage-Nassar G, Rotterdam H, Frank D, et al. Esophagitis dissecans superficialis associated with celiac disease. Gastrointest Endosc. 2003; 57:140-1.

42. Morgenstern M. Tube-like expulsion of esophageal mucosa (esophagitis exfoliativasive dissecans superficialis). Zentralbl Allg Pathol 1952;89: 17-22.

43. Kurian A, Haber R. Methotrexate-induced cutaneous ulcers in a nonpsoriatic patient: case report and review of the literature. J Cutan Med Surg. 2011;15:275-9.

44. Albert DM, Ally MR, Moawad FJ. The sloughing esophagus: a report of five cases. Am J Gastroenterol. 2013;108(11), 1816-7.

45. Brandt L. Esophagitis dissecans superficialis. Gastrointest Endosc. 2011; $74: 403-4$ 